\title{
Black Cataract and SICS: A Safe Surgery with Excellent Outcome
}

\author{
Prasanth Gireesh $^{1 *}$, Abdulmajeed Alharbi ${ }^{2}$ and Poulomi Das ${ }^{3}$ \\ ${ }^{1}$ Medical Consultant, M.N. Eye Hospital, Chennai, India \\ ${ }^{2}$ Department of Ophthalmology, College of Medicine, Qassim \\ University, Saudi Arabia \\ ${ }^{3}$ Fellowship in Cataract and IOL, Aravind Eye Hospital, Pondicherry, India
}

*Corresponding Author: Prasanth Gireesh, Medical Consultant, M.N. Eye Hospital,

Chennai, India.
Received: March 30, 2020

Published: April 21, 2020

(C) All rights are reserved by Prasanth

Gireesh., et al.

\begin{abstract}
Black cataract is quite common in developing country like India. The biggest problem while dealing with these is, the nucleus is quite hard to break by phaco energy. As a result, post op Striate keratitis is very common. Along with intra op complications are also pretty high for these. On the other hand, SICS is quiet safe surgery when dealing with intra op and post op complications. Here, we are reporting a case report of, how black cataracts can be safely removed by SICS technique. Both intra op and post op complications are less here compared to phaco. Though chances of healing of wound will take a few days longer compared to phaco, but we believe, the odd s are favorable here for SICS surgery.

Keywords: Black Cataract; SICS Surgery
\end{abstract}

\section{Introduction and Case Report}

Black cataract always poses challenge to the surgeons. The large nucleus is not only difficult to phaco emulsify but also make the zonules weak leading to chances of ZD more common. Sometimes they are associated with Pseudoexfoliated material which makes the scenario more scary for the operating surgeon. In developing country like India, its not a very rare condition facing on OT table. The problem here is to continue the case with phaco or convert the case to SICS. Phaco surgery in black cataracts needs a lot of phaco energy. Sometimes post-operative outcome becomes bad because of corneal edema [1]. These oedema takes few weeks to subside as well. So, despite a good work of making a small incision to emulsify the nucleus and put a foldable lens, the outcome may not be as expected. The situation goes out of hand when the zonules are weak or PC rent happen during the surgery. These cataracts are notorious for creating on table complications. Managing these cases further is extremely challenging even in expert hands.

Here we are showing a simple SICS technique to remove black cataracts with out and complications and implant a lens in bag safely.

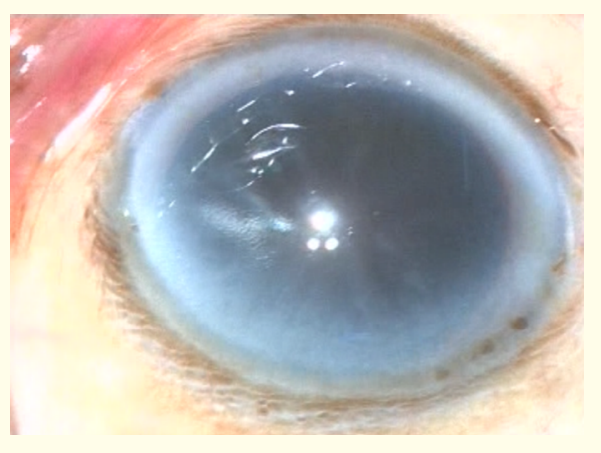

Figure 1: On table figure of Black cataract under microscope.

\section{Incision and sclero corneal tunnel}

After conjunctival peritomy in superior or supero temporal quadrant, a 6 - $6.5 \mathrm{~mm}$ frawn incision with 15 no blade is adequate. Depending on the size in can be extended to $7 \mathrm{~mm}$ as well. Sclero corneal tunnel made with crescent knife with inner lip little larger than the outer lip. Side pockets are better to made nicely for the nucleus to get engaged in the tunnel. This helps the tunnel to be more sealed and despite a large incision, there is no need for sutures at the end. With keratome entry is made in the centre of the tunnel and extended both sides. 


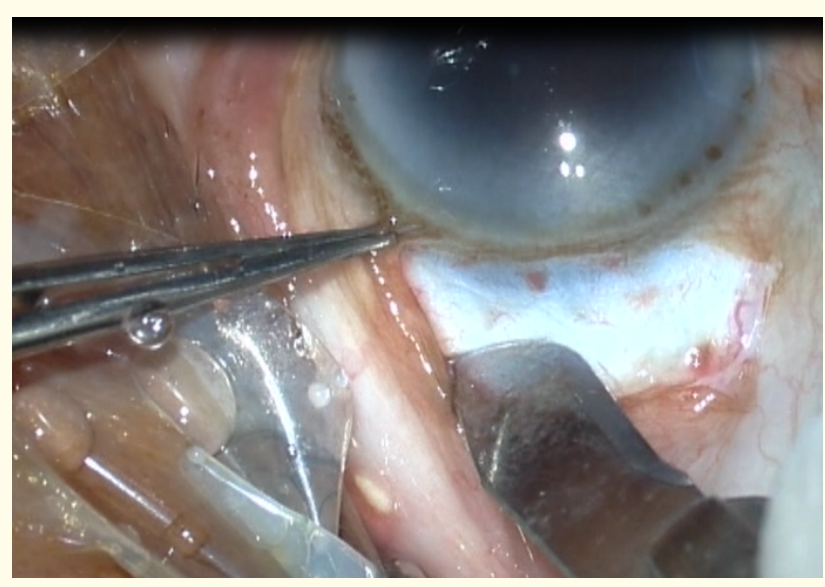

Figure 2: Incision initiated with 15 no surgical blade.

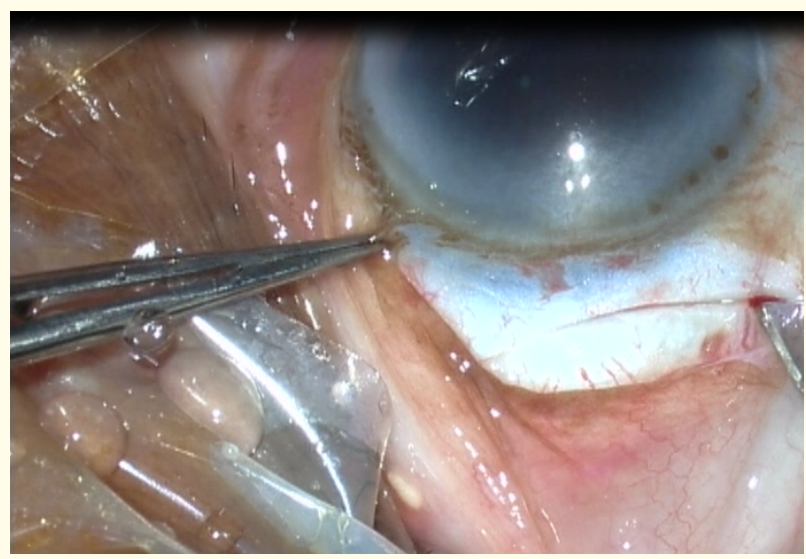

Figure 3: Full length of incision completed.

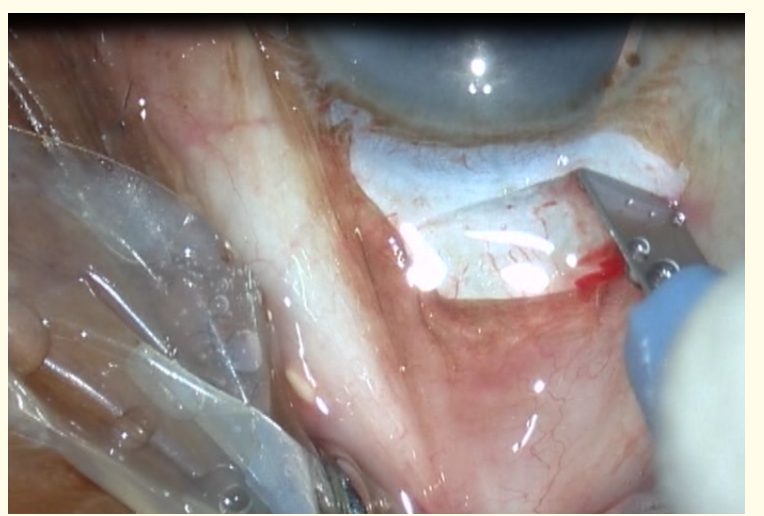

Figure 4: Making of side pockets made with crescent blade.

With Lance tip side port entry can be made before entering the anterior chamber (AC) from main wound. The side port is usually 3 clock hour away from the edge of the main wound. Trypan blue can be used to stain the capsule, as in such nucleus the retro glow of microscope do not work and visibility becomes a problem during capsulorrhexis.

\section{Capsulorrhexis}

We always recommend capsulorrhexis in all kind of cataracts. Rhexis can be done with cystitome or rhexis forceps, based on the surgeon preference. Here we are using a cystitome to initiate and complete the whole rhexis. If rhexis run off, despite all efforts, surgeon can always convert to canopener as it is more safer in these kind of nucleus. Double rhexis or c-shaped rhexis are also a good options to complete rhexis successfully $[2,3]$.

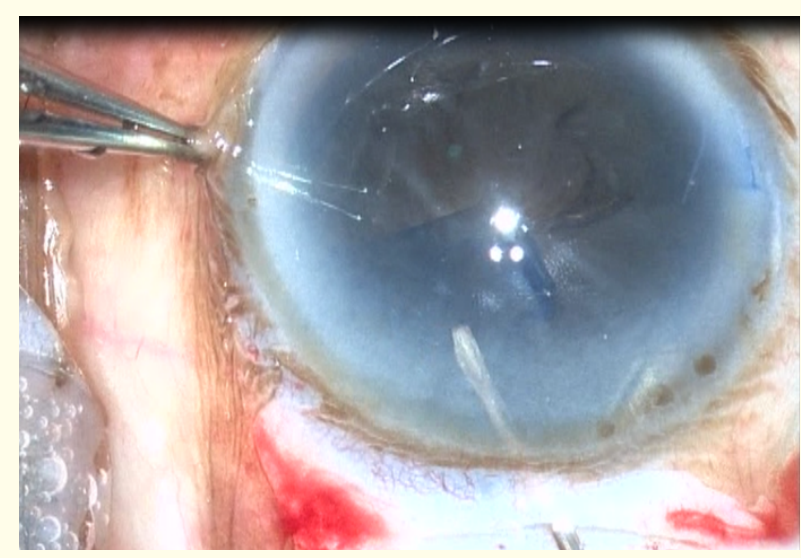

Figure 5: Capsulorrhexis with cystitome.

\section{Hydrodissection}

Controlled hydrodissection can always be tried. Vigorous hydrodissection is of course not recommended here. Due to large nucleus, usually nucleus prolapse do not happen during hydrodissection.

Bimanual technique is a safer technique when we know we have completed a complete hydrodissection and nucleus is freely moving in the bag. Otherwise, chances of zonular dialysis or complete whole bag removal during nucleus delivery is possible.

Nucleus delivery and cortical wash

Visco delivery or Vectis delivery of nucleus can be done. Despite a black cataract, cortical matters will be remaining. And the are notorious for creating PCO later. Careful cortical wash with simco with pulling the cortex in clockwise and anti clockwise manner actually poses less strain on the zonules. Its always ideal to do a proper cortical clean ups. 


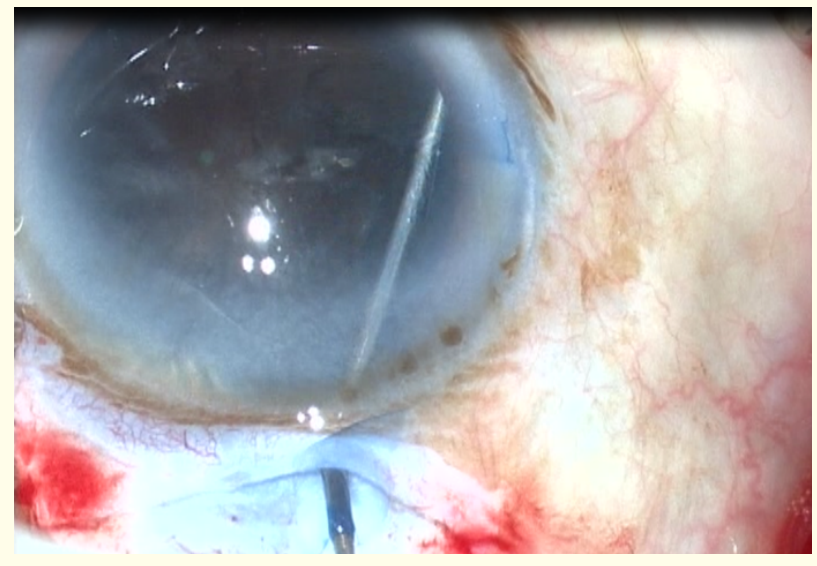

Figure 6: Hydrodissection being done.

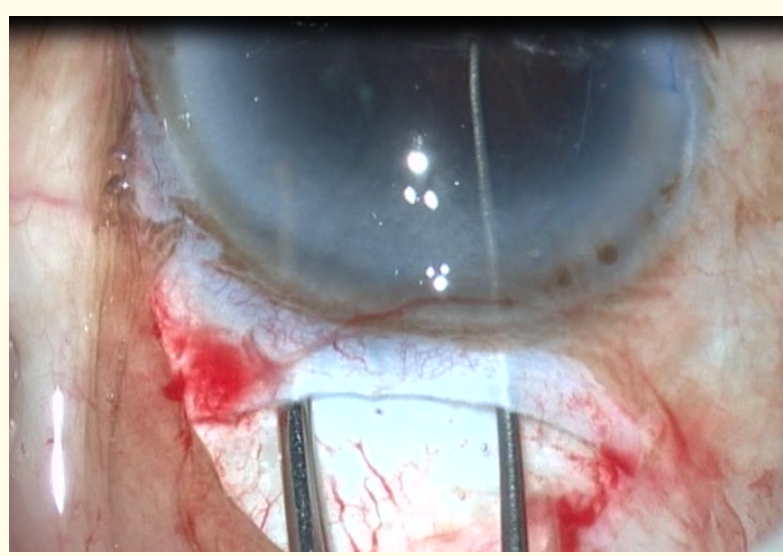

Figure 7: Bimanual prolapse of nucleus on process.

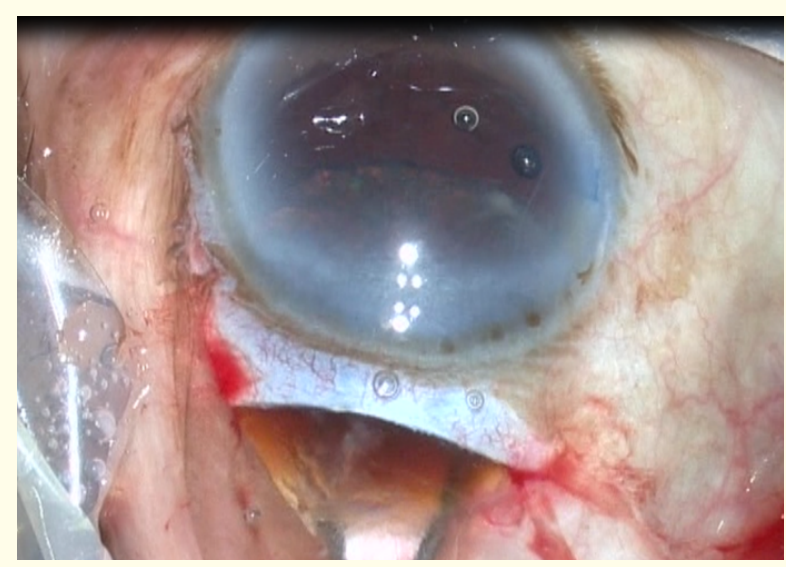

Figure 8: Nucleus being delivered with vectis.

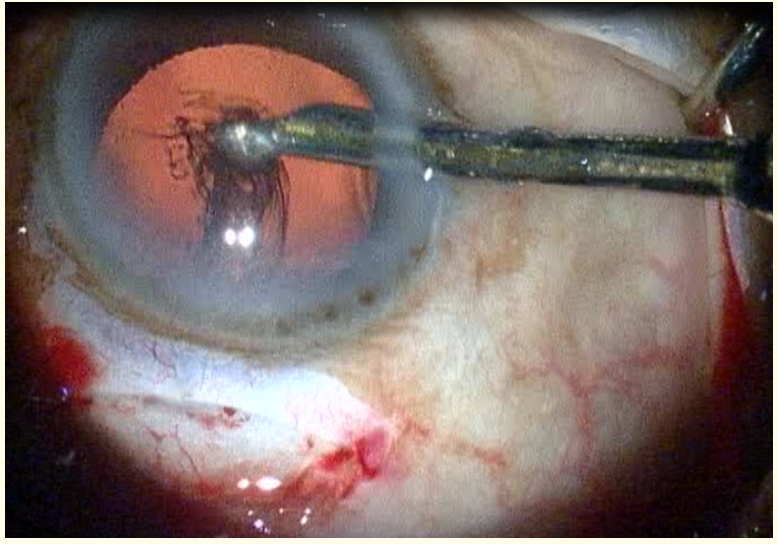

Figure 9: Cortical wash under retroillumination.

Lens

Based on pc status any lens can be placed. Here we are using 3 piece PMMA lens. With lens holding forceps, lens is placed in the bag under viscoelastic materials. Once the lens is placed visco can be cleaned up from AC and also from behind the lens with the help of simco cannula.

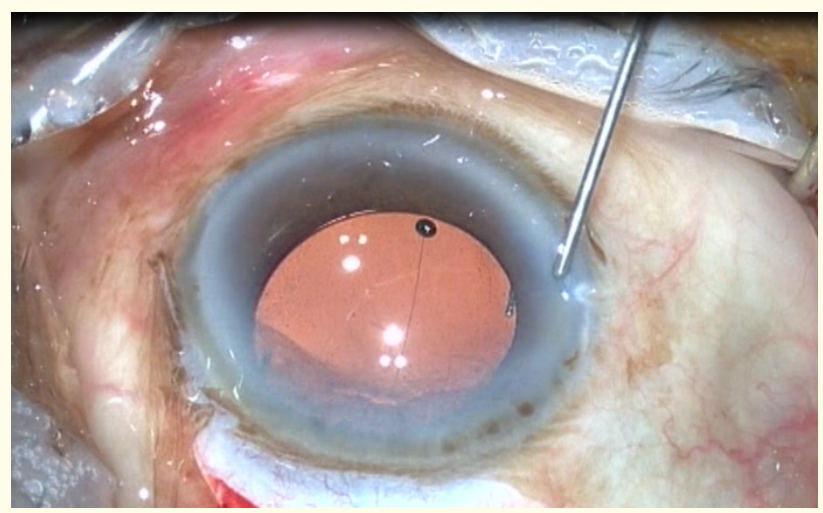

Figure 10: IOL nicely centered in bag.

\section{Discussion}

A large adequate tunnel with 6 - $7 \mathrm{~mm}$ rhexis is most essential for safe SICS in black cataracts. All it needs is the expertise of the surgeon in making a proper 3 planner tunnel and surgeon must be vigilant while performing all the steps to detect any abnormality before hand. Rhexis run off can be possible with PC rent but all these complications can be handled easily. Post op outcome of such cases are no doubt comparable to phaco as well. With a complete round rhexis placing foldable IOLs are not an issue. As a result any variety of lenses can be used here as well following SICS. 


\section{Conclusion}

In old patients with decreased endothelial cell count, SICS is a blessing compared to phaco. The incision site can always be placed based on the astigmatism of the eye. Even temporal SICS can be done in such cases if surgeon is expert in good tunnel making.

\section{Bibliography}

1. Chakrabarti A and Singh R. "Phacoemulsification in eyes with white cataract". Journal of Cataract and Refractive Surgery 26.7 (2000): 1041-1047.

2. Vasavada A and Singh R. "Surgical techniques for difficult cataracts". Current Opinion in Ophthalmology 10.1 (1999): 46-52.

3. Gimbel HV and Willerscheidt AB. "What to do with limited view: the intumescent cataract". Journal of Cataract and Refractive Surgery 19.5 (1993): 657-661.

\section{Assets from publication with us}

- Prompt Acknowledgement after receiving the article

- Thorough Double blinded peer review

- Rapid Publication

- Issue of Publication Certificate

- High visibility of your Published work

Website: $\underline{w w w . a c t a s c i e n t i f i c . c o m / ~}$

Submit Article: www.actascientific.com/submission.php

Email us: editor@actascientific.com

Contact us: +919182824667 